Rev. High Pressure Sci. Technol., Vol. 7 (1998) 1429 1431

\title{
Supercritical Water Cracking and Washing of Used Polymer
}

\author{
T. Tennoh, H. Enomoto*, C. Wakai ${ }^{\dagger}$, and M. Nakahara ${ }^{\dagger}$ \\ Nishikawa Rubber Co., Ltd., Nishi-ku, Hiroshima 733, Japan \\ *Department of Geoscience and Technology, Faculty of Engineering, Tohoku University, Aoba-ku, Sendai 980-77, Japan \\ ${ }^{\dagger}$ Institute for Chemical Research, Kyoto University, Uji, Kyoto 611, Japan
}

\begin{abstract}
A rubber polymer (EPDM, ethylene-propylene-diene-terpolymer) and its vulcanized rubber were cracked to paraffin oil (PO) in NaOH-containing supercrtitical water (SCW) at $420^{\circ} \mathrm{C}$ and $30 \mathrm{MPa}$. PO recovered as a hexane-soluble cracking component could be recycled as the rubber softener because sulfurs added to the rubber for the elasticity were washed out in SCW. The SCW polymer cracking is not oxidative but reductive. The ${ }^{13} \mathrm{C}-\mathrm{NMR}$ analysis of $\mathrm{PO}$ obtained by $\mathrm{SCW}_{2} \mathrm{O}$ cracking demonstrates that the terminal methyl carbons are deuterated as a clear indication of hydrogen supply by SCW. [supercritical water, rubber, cracking, recycle, reductive]
\end{abstract}

\section{Introduction}

SCW, water at $>374^{\circ} \mathrm{C}$ and $>22.1 \mathrm{MPa}$, can be an attractive reaction field with a high kinetic energy by enabling us to attune a new reaction pathway which is not available in ambient water due to a high energy barrier, a low reactant solubility or a low molecular diffusivity [1-4]. Solubility or mixing of "hydrophobic" substances is markedly increased in SCW without a surfactant as a result of the drastic decrease in the dielectric constant [5], and the ratio of the energy barrier height to temperature can be lowered by a high temperature attainable in SCW. Chemical reaction whose energy barrier is as high as $125 \mathrm{~kJ} \mathrm{~mol}^{-1}(15000 \mathrm{~K})$ in water at $25^{\circ} \mathrm{C}$ can be accelerated by a factor of $5.4 \times 10^{11}$ at $400{ }^{\circ} \mathrm{C}$; if such a reaction is discernible on an astronomical time scale of 10 000 years in ambient water, it can be completed in one second in SCW [4]. This simple thermodynamic and kinetic consideration suggests the possibility that SCW offers an environmentally safe alternative to the use of hazardous organic solvents in various chemical processes. In this work, we attempt to disclose such a potentiality of SCW for recovering oil from polymer cracking.

The main purpose of studying how to rearrange chemical bonds between atoms by a reaction has long been confined to the development of more useful and functional materials in a more economical way. At present, furthermore, it is wanted to cultivate a new reaction path by which materials can be decomposed or transformed after their use so as to fit to the natural environment. This is important also for polymer science that has been an endeavor to synthesize polymers from monomers by mimicking and going beyond natural fibers and rubbers. Now it is necessary to study how to decompose synthetic fibers, plastics, and rubbers comsumed. As illustrated in Fig. 1, polymer such as rubber occupies a stable volley on the potential energy surface constructed by the atoms (here $\mathrm{C}$ and $\mathrm{H}$ ) constituting the monomers, and on the forward and backward sides, there exist two decomopsed states to which polymer can be driven by SCW. Of the two possible ways, one is oxidative and the other is reductive. The latter is

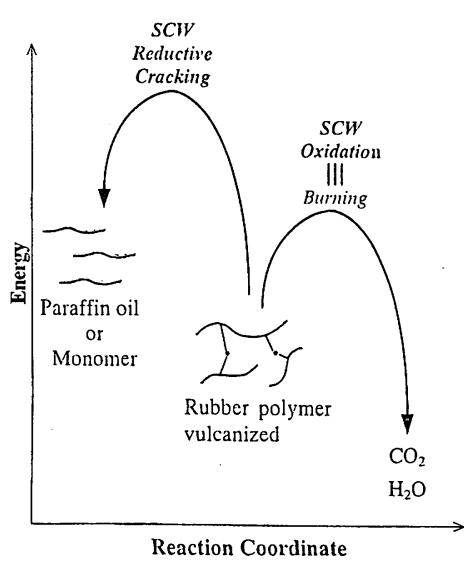

Fig.1. SCW reductive pathway vs. SCW oxidative one on an energy surface.

scarcely investigated but no doubt more desirable from the global viewpoint mentioned below.

Recently, SCW oxidative decomposition of hazardous compounds in a closed system has been explored as a new treatment method for various nature-damaging compounds containing halogen atoms [1-3]. The oxidative method leads to the thorough depletion of $\mathrm{C}-\mathrm{H}$ and $\mathrm{C}-\mathrm{C}$ bonds and the unavoidable emmission of the final product $\mathrm{CO}_{2}$ as a source of the green house warming effect. The drawback urgents us to study the SCW reductive craking of used polymers to paraffinic oil (PO) for recycle. If we can disclose a reaction pathway where hydrogen is supplied cheaply by SCW in the transformation of such wasted and nuisance materials into an intermediate form between polymeric and monomeric ones, the $\mathrm{CO}_{2}$ emmission to the atmosphere is reduced and delayed because $\mathrm{C}-\mathrm{H}$ and $\mathrm{C}-\mathrm{C}$ bonds can be regained and retained, respectively. To keep clean our earth environment, it is a promising project to crack and wash the 
vulcanized EPDM rubber to obtain useful $P O$ through a reductive SCW reaction and to elucidate the hydrogen supply mechanism by applying modern NMR spectroscopy for the interesting but complicated polymer system.

\section{Experimental}

The rubber we investigated was synthesized by adding fillers (carbon black and paraffin) and curatives (zinc oxide, sulfur, etc.) to an EPDM polymer containing ENB (2-ethylidene-5norbornene) as a diene monomer. The EPDM polymer used in this study consits of ethylene ( $68 \mathrm{~mol} \%$ ), propylene ( $29 \mathrm{~mol} \%$ ), and ENB (3 mol\%) and has a large MW (weight average, 500 000 ; number average, 80000 ). The components of the rubber are: EPDM (100), carbon black (80), paraffinic softener (60), zinc oxide (5), stearic acid (2), sulfur (2), and dibenzothiazyl disulfide (2); the parenthesized numerical values indicate the relative composition by weight. In our study of SCW cracking and washing, the rubber, its polymer or paraffin in the amount of 3-4 $\mathrm{g}$ was mixed with water $\left(\mathrm{H}_{2} \mathrm{O}\right.$ or $\left.\mathrm{D}_{2} \mathrm{O}\right)$ containing $1 \mathrm{~mol} / \mathrm{dm}^{3}$ $\mathrm{NaOH}$ in a degassed and argon-filled vessel $\left(42 \mathrm{~cm}^{3}\right)$ which is made of Hastelloy-C276. The temperature and pressure inside the vessel were monitored directly. The SCW reaction products in the liquid phase were washed with water and $n$-hexane. The PO phase has been separated from the aqueous one by $n$-hexane and dried.

\section{Results and Discussion}

As shown in Fig. 2, the rubber polymer can be cracked into PO in SCW. The molecular weight (MW) of PO, which is produced by SCW cracking and extracted by $n$-hexane after the reaction, decreases with increasing temperature. At a subcritical temperature of $350^{\circ} \mathrm{C}$ that is $24^{\circ} \mathrm{C}$ lower than the solvent critical temperature, the product has a large MW ( 100 000), which is considerably lower than that (weight average, 500 000; number average, 80000 ) of the EPDM polymer itself. At a supercritical temperature of $400^{\circ} \mathrm{C}$, a desirable cracking takes place. At a higher temperature, $420^{\circ} \mathrm{C}$, PO becomes remarkably similar to the original paraffin added as a softener to the rubber, except for a weak shoulder on the larger MW side. Thus the rubber polymer chains which are cross-linked by vulcanization can be detached and cracked into $\mathrm{PO}$, and the product $\mathrm{PO}$ has been confirmed to be useful again as a softener for the EPDM rubber synthesis. At the highest temperature $\left(450^{\circ} \mathrm{C}\right)$ studied here, the cracking proceeds to a larger extent as seen from a single peak at a lower MW position.

A maximum cracking efficiency is attained at $420^{\circ} \mathrm{C}$ where PO and the initial paraffin are almost identical with respect to MW. The yield of PO, which is defined by the weight ratio of $\mathrm{PO}$ to the sum of the EPDM and the paraffin softener, amounts to $\sim 0.9$ at this optimum temperature; most of the remainder is

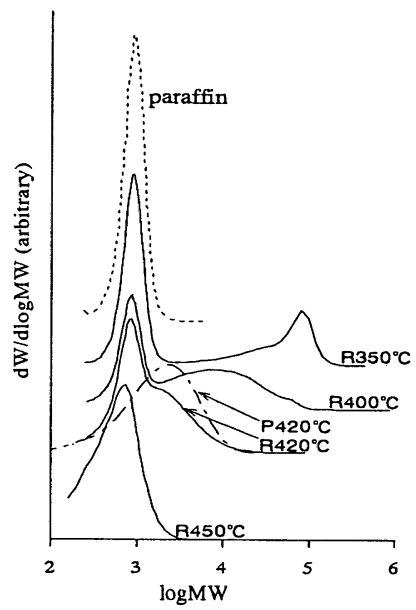

Fig.2. Molecular weight (MW) distribution of PO obtained from the vulcanized EPDM rubber and its original polymer by the SCW reaction. The ordinate $\mathrm{dW} / \mathrm{dlog} \mathrm{MW}$ indicates the probability density, and W the weight of PO. "Paraffin" indicates the paraffin softener untreated by SCW, and $350,400,420$, and $450^{\circ} \mathrm{C}$ the reaction temperatures. $R$ and $P$ denote the rubber and the polymer products at the indicated temperature, respectively. The polymer cracking in water was carried out for $30 \mathrm{~min}$ under the following temperature $(T)$ and pressure $(P)$ conditions $\left(T /{ }^{\circ} \mathrm{C}, P / \mathrm{MPa}\right)$ : $(350$ 16), $(400,26),(420,30)$, and $(450,38)$.

dissolved in water. This exceeds the formal ratio of $60 / 160=\sim 0.38$ for the rubber studied here before the SCW cracking; in the rubber, the EPDM and paraffin are mixed in a weight ratio of 100 to 60 as described above. This marked difference indicates that a large fraction of $\mathrm{PO}$ is produced through the SCW cracking. The high yield of PO with a low residual sulfur content of $<0.1 \%$ (w/w) enables us to recycle the PO obtained by the SCW cracking for an actual rubber synthesis. Without $\mathrm{NaOH}$ the sulfur content was one order of magnitude higher, and such PO prevented recycle.

To prove structural changes accompanying the SCW reductive cracking, we have above analyzed the molecular weight. As the direct evidence, however, ${ }^{13} \mathrm{C}$-NMR analysis is more powerful. To find spectral correlations, we have compared the four ${ }^{13} \mathrm{C}-\mathrm{NMR}$ spectra of the relevant compounds: the EPDM polymer, the PO, the vulcanized EPDM rubber, and the paraffin softener [6]. Based on the comparison, it has been found that the spectrum of PO was composed of the spectra of the EPDM polymer and the paraffin softener. This fact clearly demonstrates that $\mathrm{PO}$ is composed of the softener paraffin hardly attacked by SCW and a similar paraffin generated by the SCW cracking; this is consistent with the result in Fig. 2. In other words, the essential units of the rubber are retained during the SCW processing. The main $\mathrm{C}$-C-C-linkages as well as $\mathrm{CH}$ bonds are barely attacked by $\mathrm{SCW}$, and only reactive polar bonds including $\mathrm{S}$ atoms, which 
are minor in abundance but major in function in rubbers, are not randomly but selectively broken by the fairly mild reactivity of SCW.

We anticipate that if the SCW cracking is reductive, some of the new, weak peaks assignable to the methyl carbons generated by cracking are to be influenced by the addition of $D$ supplied by $\mathrm{SCW} \mathrm{D}_{2} \mathrm{O}$. Correlation of the ${ }^{13} \mathrm{C}-\mathrm{NMR}$ spectra of the PO obtained from the SCW cracking by $\mathrm{H}_{2} \mathrm{O}$ and $\mathrm{D}_{2} \mathrm{O}$ has been verified by expanding the spectra vertically to unveil hidden weak peaks among strong ones [6]. It has be seen that at least four $\mathrm{CH}_{3}$ carbons shift by $\sim 1 \mathrm{ppm}$ to a higher field and show triplet splitting due to ${ }^{13} \mathrm{C}-2 \mathrm{H}$ coupling as a result of the ${ }^{2} \mathrm{H}$ incorporation into the chain ends; the triplet splitting suggests a form of $\mathrm{CH}_{2} \mathrm{D}$. The evidence for the direct attachment of $D$ to the terminal methyl carbons confirms such a mechanism that SCW can supply hydrogen accompanying the breakage of $\mathrm{C}-\mathrm{C}$ bonds. This mechanism has been also supported the comparative ${ }^{2} \mathrm{H}$ NMR analysis.

The total yield of the product gases was $\sim 1 \%$ relative to the total weight of the initial EPDM and paraffin. By gaschromatography, the components and compositions are:

$$
\begin{gathered}
\mathrm{H}_{2}(65 \%)>\mathrm{N}_{2}(22 \%)>\mathrm{CH}_{4}(3 \%)>\mathrm{C}_{2} \mathrm{H}_{6}(2 \%) \\
=\mathrm{C}_{3} \mathrm{H}_{6}(2 \%)=\mathrm{C}_{3} \mathrm{H}_{8}(2 \%)>n-\mathrm{C}_{4} \mathrm{H}_{10}(1 \%)
\end{gathered}
$$

where the parenthesized numbers indicate the volume $\%$ of the gases under the normal conditions. A large amount of hydrogen is generated, and neither $\mathrm{CO}_{2}$ nor $\mathrm{CO}$ are detected. The excessive hydrogen in the gaseous mixture supports that the SCW cracking proceeds under not oxidative but reductive conditions.

We can recycle the PO product obtained by the reductive

\begin{tabular}{|c|c|c|c|}
\hline \multicolumn{2}{|c|}{$\begin{array}{l}\text { PO or Softener } \\
\text { Properties } \\
\end{array}$} & PO & $\begin{array}{l}\text { Paraffin } \\
\text { softener }\end{array}$ \\
\hline \multirow{4}{*}{$\begin{array}{c}\text { Mooney } \\
\text { viscometer }\end{array}$} & Viscosity, $\mathrm{ML}_{1+4}$ & 32 & 31 \\
\hline & Scorch, $\mathrm{V}_{\mathrm{m}}$ & 17 & 17 \\
\hline & $\mathrm{t}_{5}(\mathrm{~min})$ & 3.4 & 3.4 \\
\hline & ${ }^{t} \Delta 30(\mathrm{~min})$ & 3.1 & 3.0 \\
\hline \multirow{4}{*}{ Cure meter } & $\mathrm{t}^{\prime}(\mathrm{c}) 10(\mathrm{~min})$ & 0.4 & 0.4 \\
\hline & $\mathrm{t}^{\prime}(\mathrm{c}) 90(\mathrm{~min})$ & 1.9 & 1.9 \\
\hline & $\mathrm{M}_{\mathrm{L}}(\mathrm{dN} \cdot \mathrm{m})$ & 0.7 & 0.6 \\
\hline & $\mathrm{M}_{\mathrm{H}}(\mathrm{dN} \cdot \mathrm{m})$ & 7.1 & 8.2 \\
\hline \multirow{6}{*}{$\begin{array}{l}\text { Properties of } \\
\text { valcanizats }\end{array}$} & $\begin{array}{l}\text { Hardness, } \\
\text { A scale a) }\end{array}$ & 57 & 57 \\
\hline & Specific gravity & 1.1 & 1.1 \\
\hline & Tensile strength $(\mathrm{MPa})^{\text {a) }}$ & 12.2 & 12.2 \\
\hline & \begin{tabular}{|l} 
Elongation $(\%)^{\text {a) }}$ \\
\end{tabular} & 570 & 490 \\
\hline & Tear strength $(\mathrm{N} / \mathrm{m})^{\text {a) }}$ & $4.2 \times 10^{4}$ & $3.9 \times 10^{4}$ \\
\hline & Compression set $\left.(\%)^{b}\right)$ & 23 & 19 \\
\hline
\end{tabular}

Table 1. Comparison of properties of rubbers.

a) Hardness A scale, tensile properties, and compression set according to JIS K 6301. b) Compression set indicates the recovery after heating. The lower the compression set, the better the recovery.
SCW cracking as a softener. In the actual rubber synthesis, we have compared the two compounds: one contains the PO generated by a SCW reaction at $420^{\circ} \mathrm{C}$ and $30 \mathrm{MPa}$ for $10 \mathrm{~min}$, and the other contains the paraffin softener available commercially. As shown in Table 1, the properties of two compounds are almost the same except for deflected sample's recovery (compression set) and elongation. This is due to the similarity in the structure, the composition, and the molecular weight. The paraffin structure, which retains the main chain of EPDM, shows a good compatibility with the original polymer. The constituents have no functional groups oxidized and are good for the cure process. The molecular weight of the $\mathrm{PO}$ of $\sim 1000$ provides a viscosity which is suitable for the effective lubrication required for the rubber synthesis. In the industrial production of synthesis, we can blend the PO and the softener to prevent the thermal degradation of the PO. Thus, we can reuse the PO in a cyclic manner in the rubber manufacturing without strewing the oxidative forms such as $\mathrm{CO}_{2}$ and make a contribution to protect our environment.

\section{Conclusions}

Water is formed by a catalytic, photochemical or photoelectrochemical reaction between hydrogen and oxygen molecules. Although the reaction is highly irreversible under usual conditions, we can regard SCW at high enough temperatures as a source or supplier of hydrogen atoms for some chemical reactions. As a reflection of this unique feature of SCW, the vulcanized EPDM rubber and its polymer are reductively cracked in sharp contrast to the prevailing applications of SCW for oxidative decomposition of hazardous compounds. We hope that the reductive SCW cracking and washing found here take over the burning for the sake of overcoming our environmental and energy problems.

\section{References}

[1] E. Kiran and J. F. Brennecke, Eds., Supercritical Fluid Engineering Science: Fundamental and Applications, (ACS Symp. Ser. 514, American Chemical Society), Washington, DC, 1993.

[2] D.W. Tedder and F.G. Pohland, Eds., Emerging Technologies in Hazardous Waste Management III, (ACS Symp. Ser. 518, American Chemical Society), Washington, DC, 1993.

[3] L. W. Flanagin, P. B. Balbuena, K. P. Johnston, and P. J.Rossky, J. Phys. Chem., 99, 5196 (1995), and papers cited therein.

[4] M. Nakahara, in Encyclopedia of Experimental Chemistry (Jikken Kagaku Koza in Japanese), Maruzen, Tokyo (1993), 11, 149, "Very High Temperature and High Pressure Reactions".

[5] (a) M. Uematsu and E. U. Franck, J. Phys. Chem. Ref. Data, 9, 1291 (1980). (b) D. A. Archer and P. Wang, J. Phys. Chem. Ref. Data, 19, 371 (1990). (c) D. P. Fernández, Y. Mulev, A. R. H. Goodwin, and J. M. H. L. Sengers, J. Phys. Chem. Ref. Data, 24, 34 (1995).

[6] M. Nakahara, T. Tennoh, C. Wakai, E. Fujita, and H. Enomoto., Chem. Lett., No.2, 163 (1997). 